Z. Phys. Chem. 216 (2002) 575-587

(C) by Oldenbourg Wissenschaftsverlag, München

\title{
On the possibility of the Observation of Intramolecular Reaction Dynamics in Liquids by Time-resolved X-ray Scattering
}

\author{
By S. Techert ${ }^{1, *}$ and S. Schmatz ${ }^{2}$ \\ ${ }^{1}$ Max-Planck-Institut für Biophysikalische Chemie, Abt. 010, D-37070 Göttingen, \\ Germany \\ ${ }^{2}$ Institut für Physikalische Chemie der Georg-August-Universität Göttingen, \\ Tammannstr. 6, D-37077 Göttingen, Germany
}

Dedicated to Prof. Dr. Dr. h.c. mult. Jürgen Troe

on the occasion of his 60th birthday

(Received August 20, 2000; accepted March 30, 2001)

\section{Time-resolved X-ray Diffraction / Synchrotron Radiation / Large Amplitude Motion / Isomerisation Reactions}

The feasibility of time-resolved structural studies using pulsed X-ray radiation from a 3rd generation synchrotron is discussed and compared with scattering experiments which might be possible with a future free electron laser (X-FEL). As an example, it is shown that the structural changes during the isomerisation process of trans-stilbene into cis-stilbene can be observed using time-resolved X-ray diffraction in the gas-phase as well as in solution.

\section{Introduction}

In this article, the possibility of time-resolved molecular structure studies using pulsed X-ray radiation from a synchrotron of the 3rd generation is discussed and compared with scattering experiments which might be performed with a future free electron laser (X-FEL) as been proposed in the context of the TESLA project at DESY in Hamburg (Germany) [1]. Pulsed X-ray radiation from an undulator at existing synchrotrons, e.g. the European Synchrotron Facility (ESRF) in Grenoble (France), the Advanced Photon Source (APS) in Chicago (USA) and SPring-8 in Harima (Japan), is a powerful new tool, which allows for tracking of structural changes during the course of chemical reactions down to the picosecond (ps) time-scale.

* Corresponding author. E-mail: stechert@gwdg.de 
The major part of these fast experiments employ the pump and probe scheme: A short laser pulse initiates the photoreaction and an X-ray pulse probes the outcome after a given delay time. The laser pulse raises the internal energy above a critical threshold and initiates the reaction. The structural evolution of the photo-triggered reaction is then followed by a sequence of static data sets at different points of time, each representing a snapshot at a given time in the course of the reaction.

In 1995, the first experiment of this kind was realised at the time-resolved beamline of the ESRF [2,3]. Here, the structural dynamics of the photolysed myoglobin-carbonmonoxide complex was studied on the sub-microsecond time-scale. Recently, the technical time limit (50 ps) was reached through implementation of a femtosecond (fs) laser as pump source, which was phaselocked to the X-ray pulses [4]. Due to the high repetition frequency of the laser and the X-rays $(896.6 \mathrm{~Hz})$ monochromatic experiments are possible, which require a stroboscopic sampling strategy. Using the apparatus described elsewhere $[4,5]$ and applying this method to chemical systems, the transient intramolecular reorganisation processes in solid $\mathrm{N}, \mathrm{N}$-dimethylaminobenzonitrile were studied on the ps time-scale [5,6]. In the liquid phase, the light-excited reaction intermediates of the unimolecular dissociation of molecular iodine in $\mathrm{CH}_{2} \mathrm{Cl}_{2}$ were probed (also on the ps time-scale) [7,8].

The focus of this article is the question as to whether light-triggered largeamplitude motions like the trans/cis-isomerisation of trans-stilbene can be probed with state-of-the-art set-ups as the time-resolved beamline of the ESRF. Further, the feasibility of this kind of experiment is discussed for possible pump-probe experiments at an X-ray free electron laser facility (X-FEL) as planned at the Deutsches Elektronen Synchrotron (DESY) [1,9-11].

The largest light-triggered intramolecular motion which can be thought of is the trans/cis-isomerisation around a double bond. Here, an example par excellence is the isomerisation of trans-stilbene to a mixture of cis- and trans-stilbene [12-16]. The simplified reaction scheme of the photocycle of trans-stilbene is displayed graphically in Fig. 1. After the electronic excitation of trans-stilbene (1) through the absorption of a laser pulse, the Franck-Condon state (2) is generated. Due to strong repulsive interaction the system relaxes within some ps via the so-called phantom state $(\mathbf{3})$ to the cis- and trans-products of the electronic ground state (1 and 4) [17, 18].

Experimentally, the radiationless isomerisation reaction can be probed by (ultra-)fast quenching of the fluorescence of trans-stilbene. The characteristic (quenched) fluorescence varies with the experimental conditions (e.g. gas-jet experiments, pressure-experiments in the liquid phase or studies on stilbene derivatives) between femtoseconds and some picoseconds [19-30]. The reaction scheme is complicated by the fact that the formed cis-product can also be excited in the next step of a repetitive experiment (not shown in Fig. 1) [31-34]. Starting the reaction from the cis-conformer dihydrophenanthrene (DHP)/phenanthrene might be formed [33]. 
Most important for the present work - and this is the reason why e.g. state (3), where the phenyl groups are tilted $90^{\circ}$ against each other, is mentioned are the configurational changes during the isomerisation reaction.

\section{Simulation of the diffuse $X$-ray signal}

A theoretical description of probing an isomerisation process by X-ray scattering starts with a quasi-stationary treatment of the various possible conformations sketched in Figs. 1 and 2.

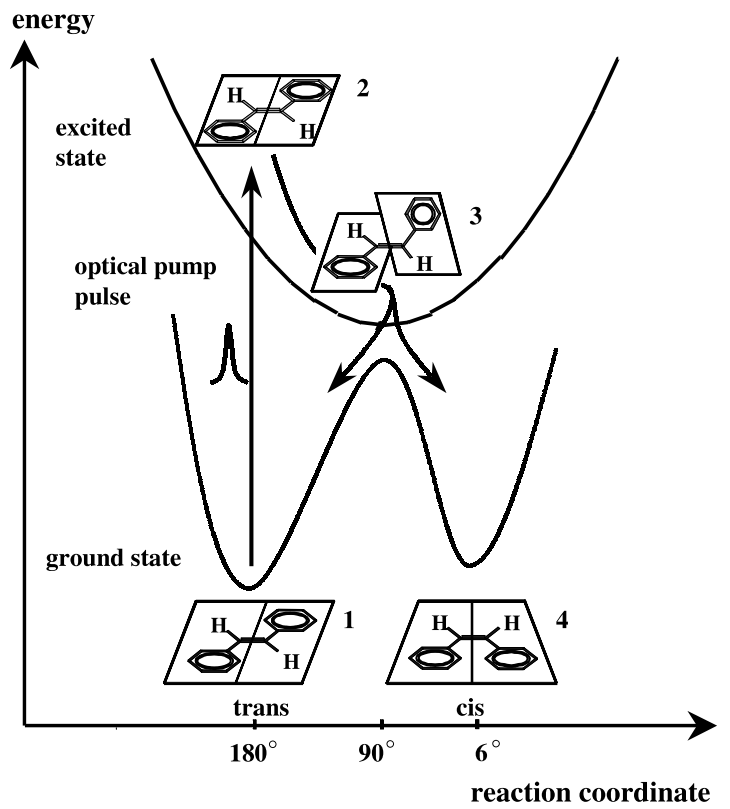

Fig. 1. Simplified reaction scheme of the trans/cis-isomerisation of stilbene.

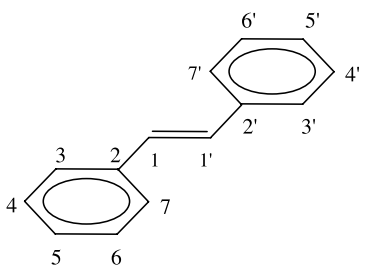

1

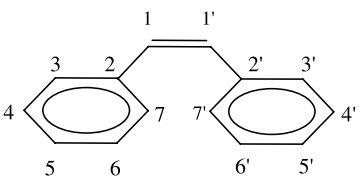

4

Fig. 2. Assignment of the atoms in trans-(1) and cis-stilbene (4). 


\subsection{Simulation of a gaseous sample}

Applying the Debye scattering equation for diffuse scattering of a gaseous sample [35], it is possible to derive an analytical expression for the change of the diffracted intensity as a function of the torsional angle which describes the motion around the ethylenic double bond (in e.u.):

$$
\begin{aligned}
I= & \sum_{u} \sum_{v} f_{u} f_{v} \sin \left[4 \pi q r_{u v}\right] /\left[4 \pi q r_{u v}\right] \\
= & \sum_{m} \sum_{m^{\prime}} f_{m} f_{m^{\prime}} \sin \left[4 \pi q r_{m m^{\prime}}\right] /\left[4 \pi q r_{m m^{\prime}}\right] \\
& +\sum_{n} \sum_{n^{\prime}} f_{n} f_{n^{\prime}} \sin \left[4 \pi q r_{n n^{\prime}}(\vartheta)\right] /\left[4 \pi q r_{n n^{\prime}}(\vartheta)\right] .
\end{aligned}
$$

Here, $\vartheta$ is the torsional angle and $q$ is defined as $q=\sin \Theta / \lambda$; $\Theta$ denotes the Bragg angle of the diffracted X-ray beam and $\lambda$ is the $\mathrm{X}$-ray wavelength. The indices $u$ and $v$ denote the atoms of an arbitrary molecule.

In stilbene, the $r_{m m^{\prime}}$ denote all bond distances between two carbon atoms $m$ and $m^{\prime}$, which are not affected by the torsional motion around the ethylenic double bond. The interatomic distances which change during the isomerisation are described by the $r_{n n^{\prime}}(\vartheta)$; these are all distances between the carbon atoms $n=2, \ldots, 7$ of the first phenyl moiety to the carbon atoms $n^{\prime}=2^{\prime}, \ldots, 7^{\prime}$ of the second phenyl moiety (see Fig. 2). E.g., the exact changing of the distance between $\mathrm{C}(2)$ and $\mathrm{C}\left(2^{\prime}\right)$ during the isomerisation is described by:

$$
r_{22^{\prime}}(\vartheta)=\sqrt{ }\left[k_{1}-k_{2} \cos \vartheta\right]
$$

with

$$
\begin{aligned}
& k_{1}=4 r_{12}^{2}+r_{11^{\prime}}^{2}-4 r_{12} r_{11^{\prime}} \cos \left(\alpha_{211^{\prime}}\right)-2 r^{2}{ }_{12} \sin ^{2}\left(\alpha_{211^{\prime}}\right) \\
& k_{2}=2 r^{2}{ }_{12} \sin ^{2}\left(\alpha_{211^{\prime}}\right),
\end{aligned}
$$

where $r_{12}$ is the distance between $\mathrm{C}(1)$ and $\mathrm{C}(2)$. Due to the symmetry of stilbene, it holds $r_{12}=r_{1^{\prime} 2^{\prime}}$. The distance between $\mathrm{C}(1)$ and $\mathrm{C}\left(1^{\prime}\right)$ is denoted by $r_{11^{\prime}}$, and $\alpha_{211^{\prime}}$ is the angle between the $\mathrm{C}$ atoms of the ethylenic double bond and $\mathrm{C}(2)$. Using (2) in the cis-conformation $(\vartheta \cong 0)$, one obtains $r_{22^{\prime}}^{\text {cis }}=\sqrt{ }\left[k_{1}-k_{2}\right]$, whereas in the trans-conformation $(\vartheta=\pi)$ the distance is given by $r_{22^{\prime}}^{\text {trans }}(\vartheta)=\sqrt{ }\left[k_{1}+k_{2}\right]$.

Fig. 3 shows the simulated change of the absolute scattered intensity of gaseous stilbene according to (1) as a function of $q$ and $\vartheta$. The torsional angle $\vartheta$ was fixed in the calculations, whereas all other geometrical parameters were optimised with respect to energy. The described procedure has the advantage that other large amplitude motions, e.g. the tilting between the phenyl and the ethylene moieties, which can also change during the isomerisation process, are taken into account $[36,37]$. Since for the explorative calculations in this work no high-level ab initio computations are required we decided to make use of a semi-empirical method, viz. AM1. The program package GAUSSIAN 


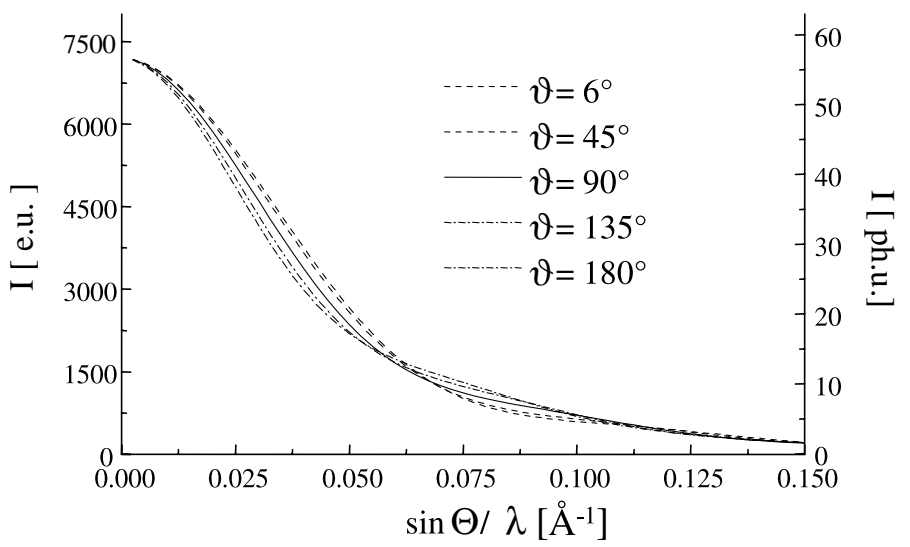

Fig. 3. The diffuse scattering of gaseous stilbene as a function of $l=\sin \Theta / \lambda$ for different torsional angles $\vartheta$ according to the Debye scattering equation.

98 was used throughout [38]. In order to assess the quality of these computations, we performed calculations on the potential energy hypersurface (PES) of the electronic ground state using the density functional (DFT) variant B3LYP in conjunction with the $6-311+\mathrm{G}^{* *}$ basis set. Overall, the calculated geometrical parameters, even those pertinent to large amplitude motion, are in good agreement with the results obtained employing AM1. E.g., the torsional angle with respect to the ethylenic double bond was calculated to be $6.4^{\circ}$ (B3LYP/6$311+\mathrm{G}^{* *}$ ) and $3.1^{\circ}$ (AM1). The AM1 calculations on the PES of the first exited electronic state were performed in the interval $0^{\circ} \leq \vartheta \leq 180^{\circ}$ in steps of $5^{\circ}$. In future work, more sophisticated electronic structure calculations will be considered. Here, only the demonstration of the general feasibility is concerned.

The calculations were performed assuming $30 \%$ of photo-excitation, a decay of $98 \%$ of the photo-activated species via the phantom state and a fluorescence from the Franck-Condon state of $2 \%$. Thus, competitive photo-reactions besides fluorescence and isomerisation are neglected. Fig. 3 shows the clear modulation of the intensity signal as a function of $q$ for the different diastereomeres, in particular against the $90^{\circ}$-conformation as reference. In this conformation, both phenyl moieties stay perpendicular to each other. The $6^{\circ}$ and $45^{\circ}$-conformations show opposite intensity behaviour with respect to the $90^{\circ}$-conformation compared to the $135^{\circ}$ - and $180^{\circ}$-conformation (e.g., intensity increase at $q<0.060 \AA^{-1}$ instead of intensity decrease).

Fig. 4 shows the intensity change with respect to the trans-educt. The relative intensity change $\Delta I / I$ is plotted as a function of $q$ for different values of $\vartheta$. Trans-stilbene $\left(\vartheta=180^{\circ}\right)$ was chosen as reference. Going from $180^{\circ}$ to $6^{\circ}$, the relative peak maxima are shifted to larger $q$, and the change in intensity is increased at lower $q$. The shift of the relative peak maxima also increases with $q$. 


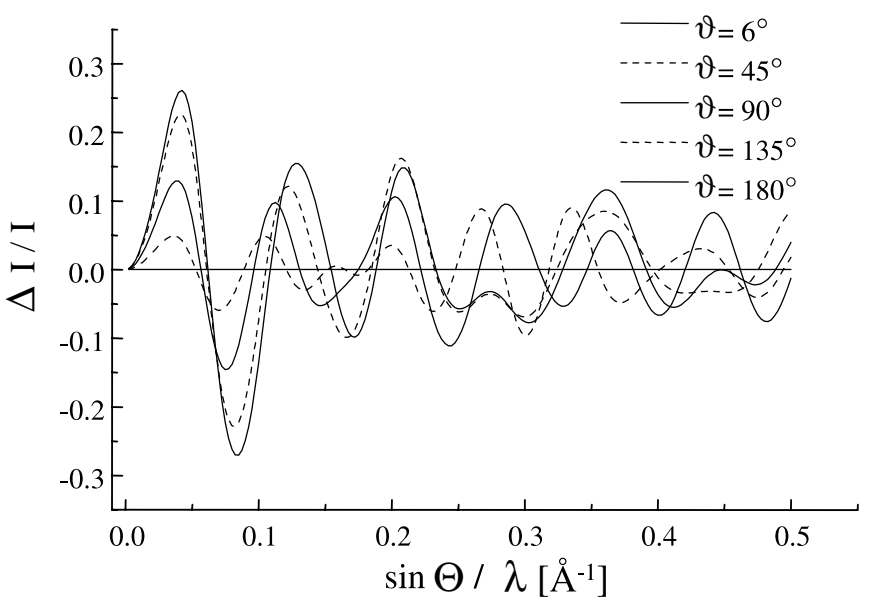

Fig. 4. Change of the diffuse scattering of gaseous stilbene during the photo-induced isomerisation. A photo-excitation level of $30 \%$ was assumed.

In order to find the optimum X-ray wavelength for monochromatic data sampling which can probe the isomerisation, a compromise between the intensity change and the shift of the diffraction maxima has to be found: Since the effect of the maxima shift increases with $q$ whereas the intensity change decreases, the X-ray wavelength has to be chosen in such a way that for a selected $q$ range the intensity change and the shift of the Bragg maxima are optimised with respect to each other. According to Fig. 4 this should be valid in the gas phase for $0.03 \AA^{-1} \leq q \leq 0.3 \AA^{-1}$, which is larger than the usable range for the diffuse scattering signal of stilbene in liquids (see below).

\subsection{Simulation of a diluted sample in the liquid phase}

This section describes how structural changes, e.g. isomerisation processes, can be probed in liquids. Fig. 5a and b summarise the simulated change of the scattered X-ray intensity after laser-excitation for the reactant, the phantom state and the product of stilbene dissolved in methanol. In order to reduce the dependence of the diffuse X-ray scattering to a function of $q$ (and $\vartheta$ ) only, in a first approximation gas-like behaviour of diluted stilbene solution is assumed. In future work, this approach will be corrected for the solvent-solute interaction, which is mainly determined by higher order terms in the diffraction intensity.

Intermolecular interactions for the solvent are taken into account, because the used X-ray diffraction signal of methanol is a measured signal [4]. The influence of intermolecular forces results mainly in a decrease of the scattered $\mathrm{X}$-ray intensity and a shift of the maxima towards smaller diffraction angles compared to the gas-phase simulation (Fig. 4). As in Sect. 2.1, it is assumed 


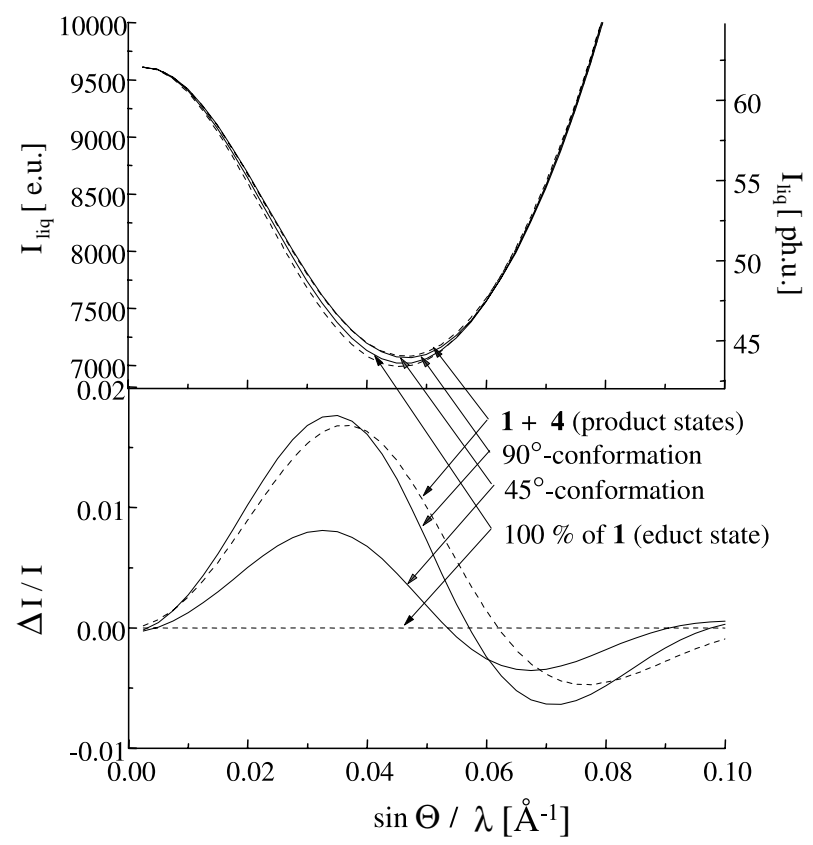

Fig. 5. Absolute (a) and relative (b) diffuse scattering signal of the photo-induced isomerisation of stilbene in methanol taking a concentration of $5 \mathrm{mM}$ trans-stilbene. A photoexcitation level of $30 \%$, a population of the phantom state of $98 \%$ and back-fluorescence from the Franck-Condon state of $2 \%$ were assumed.

that $30 \%$ of the species are photo-excited; $98 \%$ of them decay via the phantom state and $2 \%$ fluoresce back from the Franck-Condon state. Reference signal is the reactant signal of $5 \mathrm{mM}$ trans-stilbene solution in methanol.

Due to the dominant methanol signal the $q$ interval with the biggest signal change ranges from $q=0 \AA^{-1}$ to $0.15 \AA^{-1}$ which is smaller than the usuable range in the gas-phase in Fig. $4\left(0.03 \AA^{-1} \leq q \leq 0.3 \AA^{-1}\right)$. With a monochromatic X-ray energy of $E_{\mathrm{X} \text {-ray }}=11 \mathrm{keV}$, which corresponds to a wavelength of $\lambda_{\text {X-ray }}=1.127 \AA$, the range of the Bragg diffraction signal is $0^{\circ} \leq 2 \Theta \leq 19.5^{\circ}$. $E_{\mathrm{X} \text {-ray }}=11 \mathrm{keV}$ is a typical X-ray energy of an undulator device built in the storage ring of a high-energy synchrotron like the ESRF. The values are taken from [3].

Fig. $5 \mathrm{~b}$ reflects the signal decrease of the pure stilbene signal due to dilution effects by solving it in methanol. While in the gas phase (Fig. 4) the largest intensity change is $\Delta I / I=28 \%$ it decreases in the liquid phase to $\Delta I / I=2 \%$ (Fig. 5b).

Assuming an equal decay rate to the trans- and cis-product states $(1: 1)$ the intensity change, which can be expected, amounts to $1.7 \%$. The population of the $45^{\circ}$-conformation would increase the overall signal by $0.08 \%$ and the pop- 


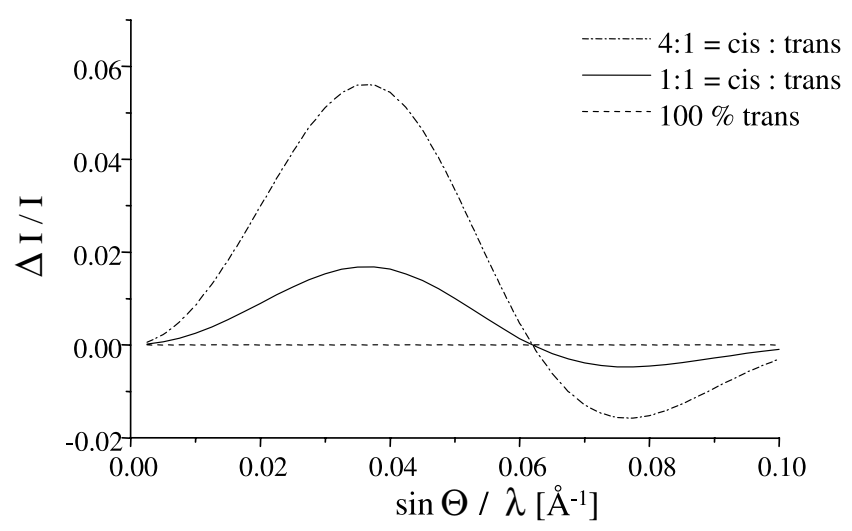

Fig. 6. Simulation of different product ratios (cis : trans-product ratio $=4: 1$ and $1: 1$ ).

ulation of the $90^{\circ}$-conformation by $1.9 \%$. More importantly, Fig. $5 \mathrm{~b}$ also shows that the change of the scattered signal occurring due to the population of the product states and due to the formation of conformational intermediates, which might be populated during the relaxation processes, is clearly distinguishable within the spatial resolution of an X-ray diffraction apparatus. Not only the change in intensity but also the phase shift of the scattered signal gives clear evidence which species is formed at a given time-delay.

In keeping with the broad diffraction signal in Fig. 5b one might argue that a change in the distribution function, e.g. between the different conformers or a change of the product state ratio $(4: 1$ instead of $1: 1)$ strongly influence the diffraction signal. As shown in Fig. 6 this is valid for the recorded intensity of the scattered signal but not for its phase. Changes in the product ratio may lead to an intensity decrease or increase but not to a phase-shift of the scattered signal. Thus, the enrichment of the cis-product due to chemical wasting will not change the signal position in $q$-space but its intensity.

\subsection{Time evolution of the scattered X-ray signal}

If one assumes that the intramolecular reaction coordinate of the isomerisation is dominated by the torsional angle $\vartheta$, in a first approximation one can assign different points of time to different geometrical conformations of stilbene. Therefore, the scattered signals of the different conformations in Figs. 3, 4 and 5 can also be regarded as the time evolution of the change in the stilbene signal during the isomerisation: At time zero all educts are in trans-conformation $\left(\vartheta=180^{\circ}\right)$, which is also the conformation of the Franck-Condon state directly after laser excitation. Some hundred fs later this state decays via several structural conformations and the phantom state $\left(\vartheta=90^{\circ}\right)$ to the trans- and cis- 
products $\left(\vartheta=180^{\circ}\right.$ and $\left.\vartheta=6^{\circ}\right)$. As shown, the phase-shift of the scattered signal mainly reflects the formation of the various conformations in time.

Distinguishing two extreme cases one can now define a time evolution of the phase-shift $2 \Theta_{\max }[t]$ and a time-evolution for the scattered X-ray signal, $\int|\Delta I| \mathrm{d}(2 \Theta)[t]$. However, both signals should reflect the ultra-fast build-up time of the several conformations and the slower decay time of 40 ps towards the trans/cis-product mixture.

\subsubsection{Conformational changes: phase-shift of the scattered signal}

Starting with e.g. the relative signal change of Fig. 5b, one can now simulate the time evolution of the maximum/minimum positions of the diffuse X-ray signal as well as the time evolution of the integrated intensity $\int|\Delta I| \mathrm{d}(2 \Theta)$ (see Fig. 7). The difference curve $\Delta I$ is defined as the signal change with respect to the diffuse scattering signal of the trans-educt $\left(t_{0}=0\right)$ :

$$
\Delta I=I(t)-I\left(t_{0}\right) .
$$

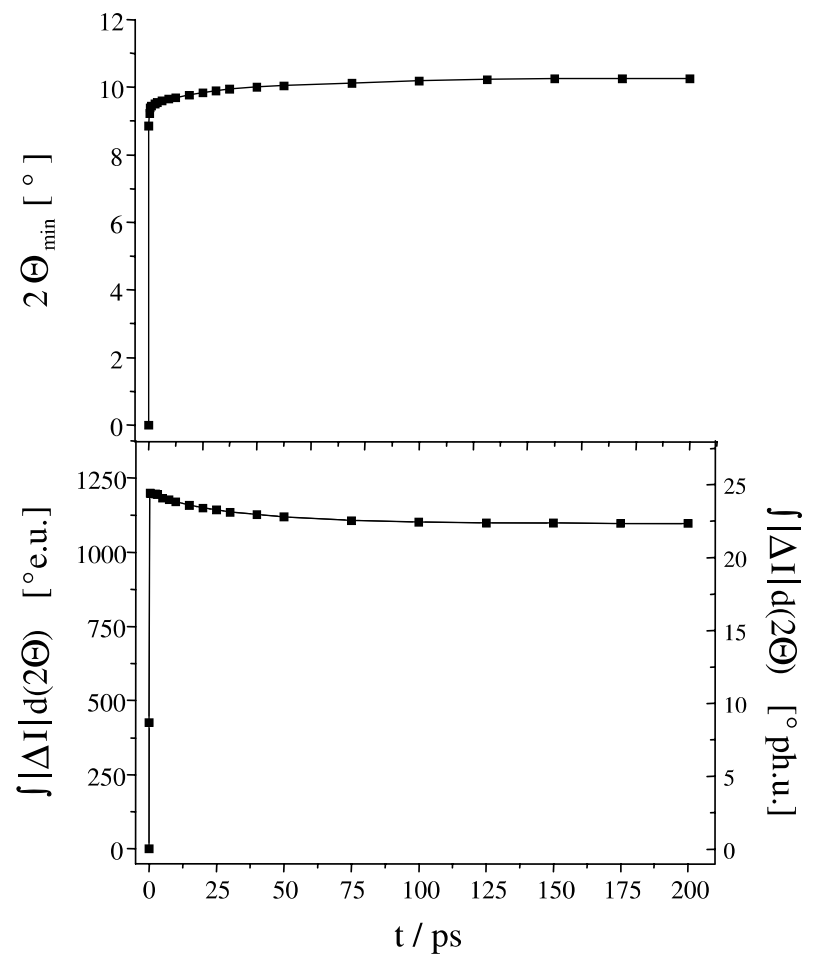

Fig. 7. Time evolution of the diffracted peak minimum at $2 \Theta_{\min } \cong 8.8^{\circ}$ (a) and the relative integrated intensity change (b) of stilbene in methanol $\left(\vartheta=45^{\circ}\right.$-conformation). 
The experiment was simulated employing a $40 \mathrm{ps}$ inverse rate constant for the isomerisation from the trans-educt via the several conformations to the trans/cis-products [27]. We assume that the lower limit for the time-scale of the conformational changes is given by the overall isomerisation rate constant.

The maximum/minimum positions of the $\Delta I$-curve were calculated as a function of $q$ for an undulator source as described in Sect. 2.2 ( $\lambda_{\mathrm{X} \text {-ray }}=$ $1.127 \AA$ ). Since the time-dependent phase-shift of the scattered signal is reflected in the position change of $2 \Theta_{\max }$ and $2 \Theta_{\min }$, the minimum at $2 \Theta_{\min } \cong 8.8^{\circ}$ was chosen as an example for this time-dependent position change. Within the time window considered, $2 \Theta_{\min } \cong 8.8^{\circ}$ follows an exponential saturation function (see Fig. 7, top):

$$
2 \Theta_{\min }(t)=A_{1}\left[1-\exp \left(-t / \tau_{1}\right)\right]+A_{2} \exp \left(-t / \tau_{2}\right)
$$

with the fitting parameters $A_{1}=10.14^{\circ}, A_{2}=-0.61^{\circ}, \tau_{1}=0.8 \mathrm{fs}$ and $\tau_{2}=$ $40 \mathrm{ps}$.

\subsubsection{Change in the distribution function: intensity increase/decrease}

As described, the time-evolution of the integrated intensity signal reflects the change in the population for a given conformation. In this - theoretical work it was considered that the build-up and decay of the different vibrational levels follow common exponential time laws. Here, build-up and decay of the $45^{\circ}$-conformation was simulated (see Fig. 7 , bottom). The integration range of the scattered signal was set to $0^{\circ} \leq 2 \Theta \leq 20^{\circ}$ :

$$
\int|\Delta I| \mathrm{d}(2 \Theta)[t]=\tilde{A}_{1}\left[1-\exp \left(-t / \tau_{1}^{\prime}\right)\right]+\tilde{A}_{2} \exp \left(-t / \tau_{2}^{\prime}\right)
$$

with $\tilde{A}_{1}=1118^{\circ}$ e.u., $\tau_{1}=1.5$ fs and $\tilde{A}_{2}=72^{\circ}$ e.u., $\tau_{2}^{\prime}=40$ ps. The fitting constants $\tau_{1}^{\prime}$ and $\tau_{2}^{\prime}$ reflect the build-up and decay time of the $45^{\circ}$-conformation.

\section{Experimental requirements}

In order to evaluate the feasibility of probing conformational changes with pulsed X-rays, several technical specifications concerning a synchrotron of the 3rd and 4th generations (X-FEL) have to be considered.

Since the expected signal changes are only weak $(0.2 \% \leq(\Delta I / I) \leq 1.8 \%)$, careful comparison between signal to noise ratio $(S / N)$ as well as simulated relative changes of intensity are required. High X-ray fluxes and signalsensitive detection methods are necessary.

Table 1 summarises the basic features concerning flux and time resolution of a synchrotron of the 3 rd generation and an X-FEL. In order to receive a diffuse X-ray signal of sufficient intensity - using a 2D CCD camera as detector $10^{7} \mathrm{X}$-ray pulses with $10^{5}$ photons/pulse have to be accumulated on the detector. Using the X-FEL as source, $1-2 \mathrm{X}$-ray pulses yield the same or an even 
Table 1. Basic features of a monochromatic X-ray beam produced in a 3rd generation synchrotron compared to a monochromatic X-ray beam of an X-FEL. $1 \AA=100 \mathrm{pm}$

\begin{tabular}{|c|c|c|c|c|c|c|c|}
\hline \multicolumn{8}{|c|}{ 3rd generation synchrotron } \\
\hline $\begin{array}{l}E_{\text {X-ray }} \\
(\mathrm{U} 20) \\
{[\mathrm{keV}]} \\
\end{array}$ & $\begin{array}{c}\lambda_{\text {X-ray }} \\
{[\AA]}\end{array}$ & $\begin{array}{l}\text { photons } / \text { pulse } \\
\text { [ph/0.1\% bw] }\end{array}$ & $\begin{array}{l}\text { focus } \\
{\left[\mu \mathrm{m}^{2}\right]}\end{array}$ & $\begin{array}{l}v_{\text {rep }} \\
{[\mathrm{Hz}]}\end{array}$ & $\begin{array}{c}\mathrm{No}_{\text {pulses }} / \\
\text { meas }\end{array}$ & $\begin{array}{c}\Delta t_{\mathrm{X} \text {-ray }} \\
{[\mathrm{fs}]}\end{array}$ & $\begin{array}{c}\Delta t_{\text {obs }} \\
\text { (Streak-cam) } \\
{[\mathrm{fs}]}\end{array}$ \\
\hline 11 & 1.13 & $10^{5}-10^{6}$ & $180 \cdot 200$ & 896.6 & $10^{7}$ & 50000 & $500-1000$ \\
\hline \multicolumn{8}{|c|}{ X-FEL } \\
\hline $\begin{array}{l}E_{\mathrm{X} \text {-ray }} \\
{[\mathrm{keV}]}\end{array}$ & $\begin{array}{c}\lambda_{\text {X-ray }} \\
{[\AA]}\end{array}$ & $\begin{array}{l}\text { photons/pulse } \\
\text { [ph/0.1\% bw] }\end{array}$ & $\begin{array}{l}\text { focus } \\
{\left[\mu \mathrm{m}^{2}\right]}\end{array}$ & $\begin{array}{c}v_{\text {rep }} \\
{[\mathrm{Hz}]}\end{array}$ & $\begin{array}{c}\mathrm{No}_{\text {pulses }} / \\
\text { meas }\end{array}$ & $\begin{array}{c}\Delta t_{\mathrm{X} \text {-ray }} \\
{[\mathrm{fs}]}\end{array}$ & \\
\hline 12.4 & 1.00 & $10^{10}$ & $10 \cdot 10$ & 5 & $1-2$ & 100 & \\
\hline
\end{tabular}

better result. Sub-ps resolution of the experimental set-up can only be obtained with a 3rd generation synchrotron (with a natural pulse width of $50 \mathrm{ps}$ ) if an IR-laser pulse triggered X-ray Streak camera is used as detection system. However, the low sensitivity of this device is not favourable for diffuse scattering experiments with in general weak signals.

An X-ray pulse generated by an X-FEL has a natural pulse-width of $100 \mathrm{fs}$ and is therefore the source of choice for structural relaxation experiments in the liquid phase.

\section{Conclusions}

In this work it could be shown that it is in principle possible to follow large amplitude motions as relaxation processes on the potential energy surface of the excited electronic state in the gas-phase as well as in the liquid phase. Provided that the isomerisation occurs on the timescale of $200 \mathrm{fs}$ to $100 \mathrm{ps}$ (or even longer), the appearance of the different conformations involved in the relaxation processes should be resolvable in the $q$-space of an X-ray scattering apparatus. However, the expectable signal change of the usually weak scattered signal is on the order of 0.2 to $2 \%$, which requires high X-ray fluxes and/or signal-sensitive detection methods.

The change in scattered signal reflects a complex superposition of intensity variations due to geometry changes during the photocycle and the change of the distribution function for different geometries at different points of time. However, it could be shown in this work that the scattered intensity variations due to a change in the distribution function can be distinguished from a scattered signal variation due to the geometrical changes. Concerning this feature of scattered diffraction signals, further effort should be spent on feasibility studies of other photo-driven reactions. 
Within the time-resolution of a synchrotron of the 3rd generation (50 ps), the ultra-fast conformational changes can only be probed if the X-ray pulses are "cut" by using an X-ray Streak camera with fs resolution [39, 40]. At an X-ray Free Electron Laser facility this problem is eliminated through the short X-ray bunch structures (100 fs).

Since with time-resolved X-ray diffraction techniques structural relaxation processes can be probed which are independent of optical selection rules, it might be possible to visualise structures and species with optically forbidden transitions including radiationless internal conversion processes.

However, even if time-resolved X-ray scattering might lead to the successful determination of ultrafast structural relaxation, it is till now not understood how coherence of motions and coherent dynamic wavepacket propagation might be reflected in these experiments. At present the latter can well be studied by different experimental approaches like coherent infrared multiphoton excitation [41].

\section{Acknowledgement}

The authors would like to thank Dr. M. Wulff for his continous interest in this work and fruitful discussions. G. Busse, M. Fechner and B. Frederichs are acknowledged for technical support of this work. The calculations were carried out at the Gesellschaft für wissenschaftliche Datenverarbeitung Göttingen (GWDG).

\section{References}

1. K. Attenkofer, P. Beaven, T. Buhrmester, M. Epple, W. Grünert, K. Von Haeften, K. Hall, J. Knoth, P. Konarev, S. Mangold, G. Materlik, A. Moelenbroek, R. Neder, J. Neuefeind, R. Neutze, H. Schwencke, S. Techert, L. Tröger, Th. Tschentscher, E. Weckert and E. Welter, in: TDR-XFEL Workshop Series, M. Epple and E. Welter, eds. (2001).

2. D. Bourgeois, T. Ursby, M. Wulff, C. Pradevand, V. Srajer, A. LeGrand, W. Schildkamp, S. Laboure, C. Rubin, T.-Y. Teng, M. Roth and K. Moffat, J. Synchr. Radiat. 3 (1996) 65.

3. V. Srajer, T.-Y. Teng, T. Ursby, C. Pradevard, Z. Ren, S. Adachi, W. Schildkamp, D. Bourgeois, M. Wulff and K. Moffat., Science 274 (1996) 1726.

4. F. Schotte, S. Techert, P. Anfinrud, V. Srajer, K. Moffat and M. Wulff, in: Synchrotron Radiation, D. Mills, ed., J. Wiley and Sons, New York (2001).

5. S. Techert, F. Schotte und M. Wulff, Phys. Rev. Lett. 86 (2001) 2030.

6. S. Techert, F. Schotte und M. Wulff, Highlights ESRF 2001 (2001) 14.

7. R. Neutze, R. Wouts, S. Techert, J. Davidson, M. Kocsis, A. Kirrander, F. Schotte and M. Wulff, submitted (2000).

8. R. Neutze, R. Wouts, S. Techert, J. Davidson, M. Kocsis, A. Kirrander, F. Schotte and M. Wulff, Highlights ESRF 2001 (2001) 15.

9. R. Brinkmann, G. Materlik, J. Rossbach and A. Wagner, Conceptual Design Report of the $500 \mathrm{GeV} e^{+} e^{-}$Linear Collider with Integrated X-ray Laser Facility, Vol. II (1999). 
10. G. Materlik and T. Wroblenwski, in: Potential Future Applications in Chemistry of an X-ray Free Electron Laser at DESY, DESY (2000).

11. Th. Tschentscher, W. Drube, H. Franz, R. Gehrke, H. Schulte-Schrepping, T. Wroblenwski and G. Materlik, in: Potential Future Applications in Chemistry of an Xray Free Electron Laser at DESY, DESY (2000).

12. J. Schroeder and J. Troe, in: Activated Barrier Crossing, G. R. Fleming and P. Haenggi, eds., World Scientific, Singapore (1993).

13. M. Sumitani, N. Nakashima, K. Yoshihara and S. Nagakura, Chem. Phys. Lett. 51 (1977) 183.

14. B. I. Greene, R. M. Hochstrasser and R. B. Weisman, J. Chem. Phys. 71 (1979) 544.

15. L. A. Brey, G. B. Schuster and H. G. Drickamer, J. Am. Chem. Soc. 101 (1979) 129.

16. J. A. Syage, W. Lambert, P. M. Felker, A. H. Zewail and R. Hochstrasser, Chem. Phys. Lett. 88 (1982) 266.

17. M. Klessinger and J. Michl, Photo Chemistry of Organic Compounds, VCH Verlagsgesellschaft, Weinheim (1990).

18. J. Troe and K.-M. Weitzel J. Chem. Phys. 88 (1988) 7030.

19. K. Yoshihara, A. Namiki, M. Sumitani and N. Nakashima, J. Chem. Phys. 71 (1979) 2892.

20. J. A. Syage, P. M. Felker and A. H. Zewail, J. Chem. Phys. 81 (1984) 4706.

21. V. Sundstroem and T. Gillbro, Chem. Phys. Lett. 109 (1984) 538.

22. J. Troe, Chem. Phys. Lett. 114 (1985) 241.

23. M. W. Balk and G. R. Fleming, J. Phys. Chem. 90 (1986) 3975.

24. J. Schroeder, D. Schwarzer, J. Troe and F. Voß, J. Chem. Phys. 93 (1990) 2393.

25. J. Schroeder, Ber. Bunsenges. Phys. Chem. 95 (1991) 233.

26. J. Schroeder, J. Troe and P. Vöhringer, Chem. Phys. Lett. 203 (1993) 255.

27. R. Mohrschladt, J. Schroeder, J. Troe and P. Vöhringer, J. Chem. Phys. 101 (1994) 7566.

28. J. Schroeder, J. Troe and P. Vöhringer, Z. Phys. Chem. 188 (1995) 287.

29. G. Gershinsky and E. Pollak, J. Chem. Phys. 108 (1998) 2756.

30. S. Schneider, B. Brem, W. Jäger, H. Rehaber, D. Lenoir and R. Frank, Chem. Phys. Lett. 308 (1999) 211.

31. S. Abrash, S. Repinec and R. M. Hochstrasser, J. Chem. Phys. 93 (1990) 1041.

32. D. C. Todd, J. M. Jean, S. J. Rosenthal, A. J. Ruggiero, D. Jang and G. R. Fleming, J. Chem. Phys. 93 (1990) 8658.

33. L. Nikowa, D. Schwarzer, J. Troe and J. Schroeder, J. Chem. Phys. 97 (1992) 4827.

34. D. C. Todd and G. R. Fleming, J. Chem. Phys. 98 (1993) 269.

35. B. E. Waren, X-ray Diffraction, Addison-Wesley Publishing Company, Reading MA (1968).

36. J. A. Syage, P. M. Felker and A. H. Zewail, J. Chem. Phys. 81 (1984) 4685.

37. J. S. Baskin, L. Banares, S. Pedersen and A. H. Zewail, J. Phys. Chem. 100 (1996) 11920.

38. Program Package GAUSSIAN 98. M. J. Frisch, G. W. Trucks, H. B. Schlegel, P. M. W. Gill, B. G. Johnson, M. A. Robb, J. R. Cheeseman, T. Keith, G. A. Petersson, J. A. Montgomery, K. Raghavachari, M. A. Al-Laham, V. G. Zakrzewski, J. V. Ortiz, J. B. Foresman, J. Cioslowski, B. B. Stefanov, A. Nanayakkara, M. Challacombe, C. Y. Peng, P. Y. Ayala, W. Chen, M. W. Wong, J. L. Andres, E. S. Replogle, R. Gomperts, R. L. Martin, D. J. Fox, J. S. Binkley, D. J. Defrees, J. Baker, J. P. Stewart, M. Head-Gordon, C. Gonzalez, and J. A. Pople, Gaussian, Inc. Carnegie Office Park, Pittsburgh PA 15106, USA.

39. G. Mourou, G. Naylor, K. Scheidt and M. Wulff, ESRF Newsletter 26 (1996).

40. K. Scheidt and G. Naylor, DIPAC, Chester UK (1999).

41. M. Quack and J. Stohner, J. Phys. Chem. 97 (1993) 12574. 